\title{
Integrated Physiological and Biochemical Assessments for the Prediction of Growth of Abdominal Aortic Aneurysms in Humans
}

\author{
Regent Lee, MBBS, MS, DPhil, FRCS, ${ }^{*} \dagger$ Philip D. Charles, PhD, $\dagger$ \\ Pierfrancesco Lapolla, ${ }^{*}$ Ismail Cassimjee, MBBCh, MMed, FCS, ${ }^{*}$ Honglei Huang, MBBS, PhD, ${ }^{\dagger} \dagger$ \\ Benedikt Kessler, PhD, $\dagger$ Roman Fischer, PhD, $\dagger$ and Ashok Handa, MD. FRCS*, on behalf of the Oxford \\ Abdominal Aortic Aneurysm Study, the Oxford Regional Vascular Services
}

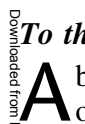
the Editor:

bdominal aortic aneurysms (AAAs) are pathological dilatations of the abdominal aorta to larger than $30 \mathrm{~mm}$ in diameter. Left 敬untreated, it eventually results in AAA rupture and high mortality. Once diagnosed, patients with AAAs are monitored by regular scans $\frac{\omega}{\omega}$ until it reaches the size threshold $(55 \mathrm{~mm}$ in diameter) deemed ongecessary for elective repair. ${ }^{1}$

Methods for the prediction of AAA growth are regarded as an Iimportant gap in existing literature according to the Vascular Surgery ف을 in the opinions of our peers. ${ }^{3}$ It can guide different aspects of clinical management in terms of the frequency of monitoring of AAAs and

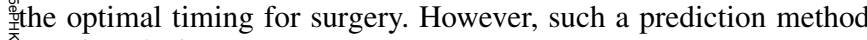

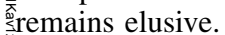

The Oxford Abdominal Aortic Aneurysm (OxAAA) Study has ofpreviously reported the importance of flow-mediated dilatation of the thbrachial artery (FMD, a physiological marker of systemic endothelial (5function) as a novel biomarker of AAA progression. ${ }^{4}$ We showed that ousing 2 variables (FMD and AAA diameter) measured at baseline, it

Fom the *Nuffield Department of Surgical Sciences, University of Oxford, Oxford, UK; and †Target Discovery Institute, Nuffield Department of Medicine, University of Oxford, Oxford, UK.

The Oxford Abdominal Aortic Aneurysm Study is supported by the following: Oxford University Hospitals NHS Foundation Trust, Jackie Walton Vascular Studies Unit (Klaus Bond et al.); University of Oxford, Medical Sciences Division Medical Research Fund (MRF/HT2016/2191); University of Oxford, Nuffield Department of Surgical Sciences; John Fell Oxford University Press Research Fund (142/075); National Institute of Health Research (NIHR) Oxford Biomedical Research Centre; British Heart Foundation Centre of Research Excellence, Oxford (RE/13/1/30181); RL is supported by a Academy of Medical Science Starter Grant, UK (SGL013/1015). PL is supported by an EU Erasmus+ traineeship studentship. BK and PDC are supported by a John Fell Oxford University Press Research Fund (133/075) and a Welcome Trust Grant (097813/11/Z). ProteinSimple (BioTechne, UK) sponsored the ELLA console for the measurement of plasma proteins.

Contributors to the OxAAA Study include: Amy Jones, Felicity Woodgate, Nicholas Killough, Kirthi Bellamkonda, Anirudh Chandrashekar; Members of the Oxford Regional Vascular Services: Chris R Darby, Alison Halliday, Linda J Hands, Dominique PJ Howard, Patrick Lintott, Tim R Magee, Andrew Northeast, Jeremy Perkins, Ediri Sideso, Emma Wilton, Ashok Handa.

The method of AAA growth prediction, as depicted by this manuscript, is covered by an international patent WO2017212210.

The authors report no conflicts of interest.

This is an open access article distributed under the Creative Commons Attribution License 4.0 (CCBY), which permits unrestricted use, distribution, and reproduction in any medium, provided the original work is properly cited.

Reprints: Regent Lee, MBBS, MS, DPhil (Oxon), FRCS (Eng), Nuffield Department of Surgical Sciences, University of Oxford, Level 6, John Radcliffe Hospital, Headington, Oxford, UK, OX3 9DU. E-mail: regent.lee@nds.ox.ac.uk.

Copyright (C) 2018 The Author(s). Published by Wolters Kluwer Health, Inc.

ISSN: 0003-4932/18/27001-00e10188

DOI: $10.1097 /$ SLA.0000000000003154 is feasible to predict the growth rate of individual patients at future 12 and 24 months with modest accuracy. ${ }^{5}$

We aim to improve the prediction algorithm by incorporating physiological (FMD, AAA size) and biochemical (circulating proteins) variables measured at the same time. In the same cohort, we utilized a proteomic method to discover circulating biomarkers that can be used to improve the prediction algorithm.

Details regarding the OxAAA study cohort and recruitment process have been published. ${ }^{5}$ In brief, this prospective study (Ethics Ref: 13/SC/0250) recruited patients who received AAA monitoring in the National Health Service setting. Baseline assessments were performed. In addition to the measurement of AAA antero-posterior diameter and FMD, fasting blood sample was collected at the same baseline appointment. Platelet-poor plasma was prepared using 2staged centrifugation as previously described ${ }^{6}$ and stored at $-80^{\circ} \mathrm{C}$ for subsequent analysis. Prospective AAA annual growth rates were calculated based on the diameter measurements in the subsequent AAA monitoring ultrasound scans. Twelve-month AAA growth data was recorded in 59 patients, with the average AP diameter of AAA of $47 \mathrm{~mm}$ at baseline. Twenty-four month growth data was recorded in 51 of these patients, as 8 of them required elective surgery by then.

We utilized a similar approach for plasma biomarker discovery as recently described. ${ }^{7}$ Based on the AAA growth rates documented during the subsequent 12 and 24 months, we selected a subset of patients for the initial discovery analysis. Plasma samples from patients with the fastest $(n=10)$ and slowest $(n=10)$ growth were pooled, then analyzed using the Proteome Profiler Antibody Arrays (R\&D Systems, \#ARY022B, \#ARY017). The array data was analyzed using the HLImage ++ software (Western Vision Software, Version PCM 18.0.7). Proteins that demonstrated at least $20 \%$ change between the 2 groups of patients were considered as potential targets with biological relevance.

For the technical validation of the protein biomarkers discovered with the above workflow, we measured the panel of proteins in individual patients using the ProteinSimple Ella enzyme immunoassay platform (BioTechne, UK). Based on the availability of the existing ELLA assays, these proteins were chosen for measurement: CXCL10, IL6, IL8, Thrombospondin, RAGE, MIP1a, MIP1b, leptin, ICAM1. Measurements from the individual patients in the discovery cohort matched the observations by the Proteome Profiler Array, with at least $20 \%$ difference observed between the fast and slow growth groups (Fig. 1A).

We tested the utility of a generalized linear model to predict growth in AAAs with an diameter between 40 and $55 \mathrm{~mm}$, as patients with an AAA in this size range are most amenable to updates in clinical practice in terms of the frequency of monitoring and the timing of elective surgery. ${ }^{4}$

We regressed the measured values of 9 proteins in combination with the measurements of AAA diameter and FMD against a 

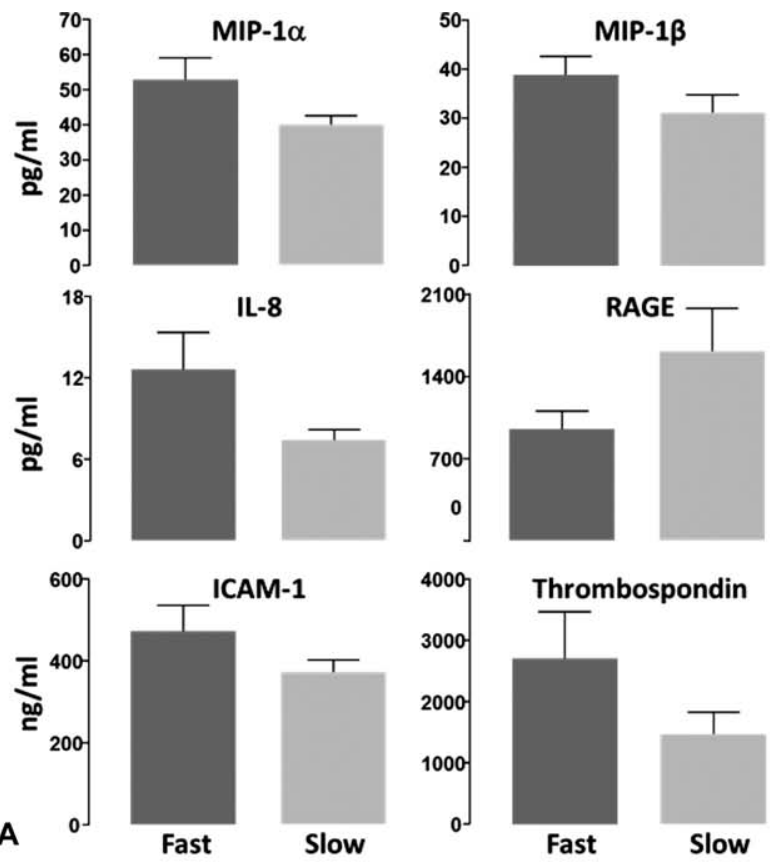

Fast
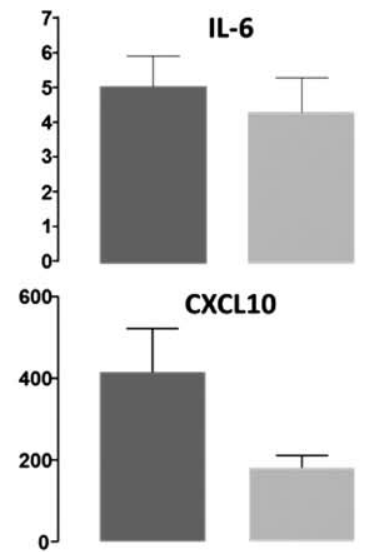

${ }_{0}^{30}{ }_{0}^{30}$

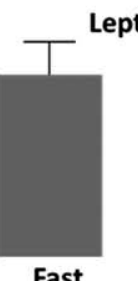

Fast

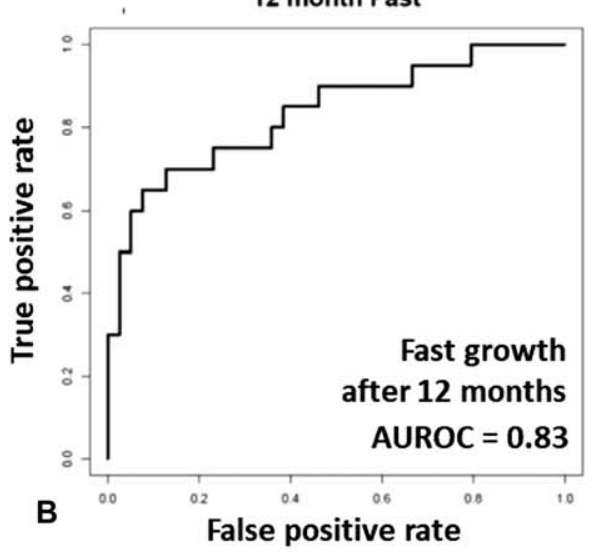

24 month Fast

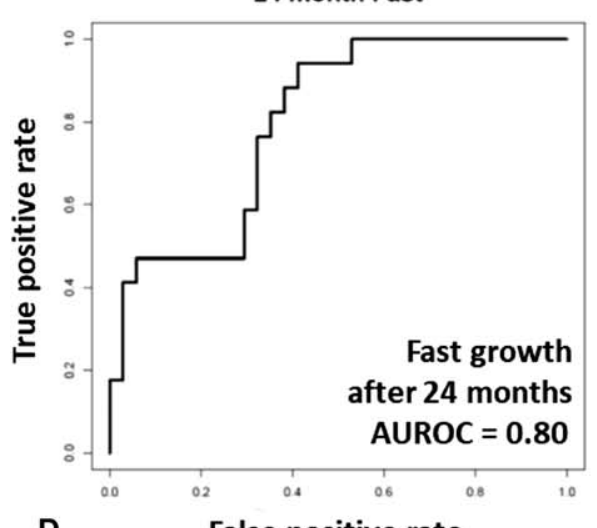

D

False positive rate
2 month Slow
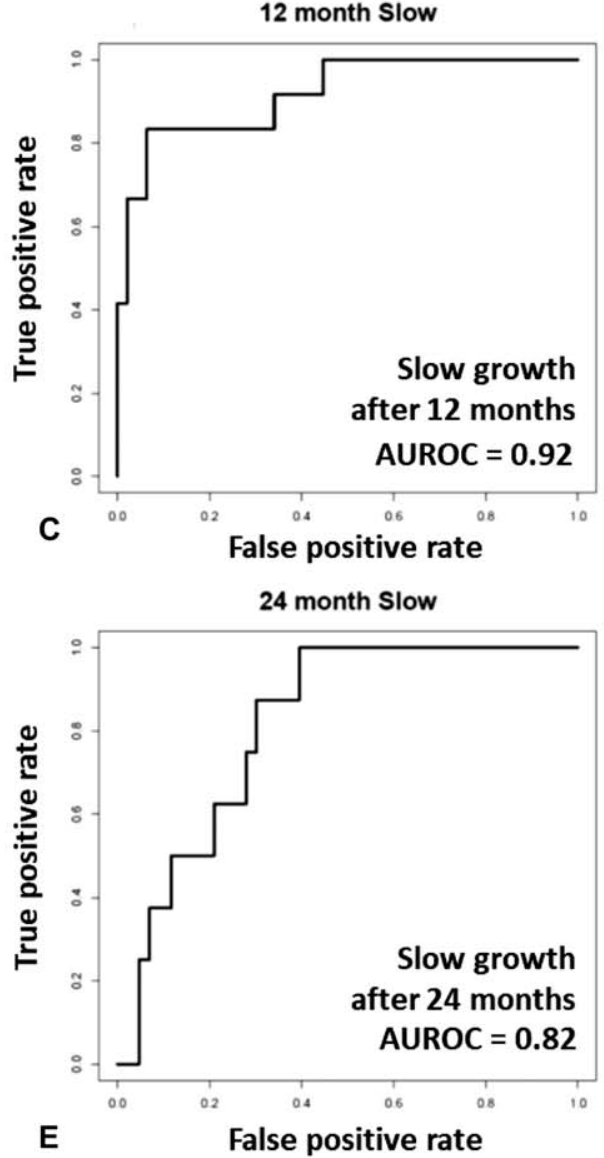

FIGURE 1. Integrated biochemical and physiological assessments for the prediction of growth of abdominal aortic aneurysm in humans. Based on the AAA growth rates documented during the subsequent 12 and 24 months, we selected a subset of patients for the initial discovery analysis. Plasma samples from patients with the fastest ( $n=10$, dark gray bars), and slowest ( $n=10$, light gray bars) growth were pooled, then analyzed using the Proteome Profiler Antibody Arrays (R\&D Systems, \#ARY022B, \#ARY017). Proteins that demonstrated at least $20 \%$ change between the 2 groups of patients were considered as potential targets with biological relevance, and measured in the individual patients using the ProteinSimple Ella enzyme immunoassay platform (BioTechne, UK). These show at least 20\% difference observed between the fast and slow growth group (A). We regressed the measured values of 9 proteins in combination with the measurements of AAA diameter and flow mediated dilatation of brachial artery (FMD, a physiological index of endothelial function) against a categorical response with levels: "Slow/no" growth (0\%), "Some" growth ( $>0 \%$ growth but still in lower twothirds of growth values) and "Fast" growth (top tertile of growth rate) for outcomes at $12 \mathrm{mo}$ and $24 \mathrm{mo}$. A multinomial logistic model was fitted using ridge regression (the regularization parameter was allowed to vary from 0 to 3 in 0.1 increments) using 10-fold cross-validation with 3 repeats to robustly estimate accuracy ( $\mathrm{R}$ V3.5.1, using glmnet and caret libraries). The final outcome models showed excellent predictive power when combined to binary comparisons of Fast versus not-Fast and Slow versus not-Slow growth. The area under receiver operating characteristics (AUROC) are 0.83 (fast growth at $12 \mathrm{mo}, \mathrm{B}$ ), 0.92 (slow growth at 12 mo, C), 0.80 (fast growth at $24 \mathrm{mo}$, D), and 0.82 (slow/no growth at 24 mo, E), respectively. 
categorical response with levels: "Slow/no" growth $(0 \%)$, "Some" growth ( $>0 \%$ growth but still in lower two-thirds of growth values) and "Fast" growth (top tertile of growth rate) for outcomes at 12 months and 24 months. A multinomial logistic model was fitted using ridge regression (the regularization parameter was allowed to vary from 0 to 3 in 0.1 increments) using 10 -fold cross-validation with 3 repeats to robustly estimate accuracy (R V3.5.1, using glmnet and caret libraries). The final outcome models showed good 3-way classification accuracy ( $73 \%$ and $58 \%$ for 12 and 24 mo respectively) and excellent predictive power when combined to binary comparisons of Fast versus not-Fast and Slow versus not-Slow growth. The area under receiver operating characteristics (AUROC) are 0.83 (Fast growth at $12 \mathrm{mo}$, Fig. 1B), 0.92 (Slow/no growth at $12 \mathrm{mo}$, Fig. $1 \mathrm{C}$ ), 0.80 (Fast growth at $24 \mathrm{mo}$, Fig. 1D), and 0.82 (Slow/no growth at 24 mo, Fig. 1E), respectively.

Cigarette smoking is a recognized risk factor in AAA pathophysiology. In addition to the measured biomarkers, we further assessed the impact of smoking history of an individual on the regression model, using pack years of smoking as a continuous variable. $((1$ pack year $=$ number of cigarettes smoked per day/20) $\times$ number of years smoked). When pack year history of smoking was included as an additional variable in the model, we observed improvements in the AUROC (fast growth at 12 mo: 0.88, slow/ no growth at $12 \mathrm{mo}: 0.95$, fast growth at $24 \mathrm{mo}: 0.81$, slow/no growth at $24 \mathrm{mo:}$ 0.82). However, in comparison with the circulating proteins and FMD (which were objectively measured by the study investigators in a prospective manner), pack years of smoking was a selfreported measurement and prone to recall/reporting bias. We have therefore primarily focused on the objectively measured biomarkers for this report.

This is the first time a study reports the utilization of a panel of circulating proteins in conjunction with a physiological measurement (FMD) to form an algorithm for the prediction of AAA growth, tested in a prospectively recruited cohort of AAA patients. To remove potential contaminations by "leakage" proteins released from residual platelets in the plasma samples, we collected platelet-poor plasma using a rigorous sample processing method. (Plasma contaminated by residual platelets will contain proteins released by platelet lysis after upon thawing of stored frozen samples. This can confound biomarker measurements in clinical studies. ${ }^{6}$ ) This maximizes the chance of plasma biomarker discovery using proteomics approach.

It is important for external cohorts to replicate the efficacy of our biomarker panel. This will require dedicated efforts to measure FMD and collect platelet-poor plasma samples in prospectively recruited AAA cohorts, as these cannot be extracted from historic studies. We hope this work serves as a primer to generate interests in the vascular surgical community and stimulates future efforts to validate the prediction algorithm.

\section{REFERENCES}

1. Chaikof EL, Dalman RL, Eskandari MK, et al. The Society for Vascular Surgery practice guidelines on the care of patients with an abdominal aortic aneurysm. J Vasc Surg. 2018;67. 2-77.e2.

2. Moll FL, Powell JT, Fraedrich G, et al. Management of abdominal aortic aneurysms clinical practice guidelines of the European society for vascular surgery. Eur J Vasc Endovasc Surg. 2011;41(suppl 1):S1-S58.

3. Lee R, Jones A, Cassimjee I, et al. International opinion on priorities in research for small abdominal aortic aneurysms and the potential path for research to impact clinical management. Int J Cardiol. 2017;245:253-255.

4. Lee R, Bellamkonda K, Jones A, et al. Flow mediated dilatation and progression of abdominal aortic aneurysms. Eur J Vasc Endovasc Surg. 2017;53:820-829.

5. Lee R, Jarchi D, Perera R, et al. Applied machine learning for the prediction of growth of abdominal aortic aneurysm in humans. EJVES Short Rep. 2018;39:24-28.

6. Lee R, Antonopoulos AS, Alexopoulou Z, et al. Artifactual elevation of plasma sCD40L by residual platelets in patients with coronary artery disease. Int $J$ Cardiol. 2013;168:1648-1650.

7. Lee R, Fischer R, Charles PD, et al. A novel workflow combining plaque imaging, plaque and plasma proteomics identifies biomarkers of human coronary atherosclerotic plaque disruption. Clin Proteomics. 2017;14:22. 